Commun. Math. Phys. 139, 215 (1991)

Communications in

Mathematical

Physics

(C) Springer-Verlag 1991

\title{
Erratum
}

\section{On the Measure of the Spectrum for the Almost Mathieu Operator}

Commun. Math. Phys. 132, 118 (1990)

\author{
J. E. Avron ${ }^{1}$, P. van Mouche $^{2}$, and B. Simon ${ }^{3}$ \\ 1 Department of Physics, Technion, Haifa, Israel \\ 2 Landbouwhogeschool Wageningen, Vakgroep Wiskunde, Wageningen, The Netherlands \\ 3 Department of Mathematics, California Institute of Technology, Pasadena, CA 91125, USA
}

Received February 8, 1991

There is a systematic factor of 2 error in the discussion and example on pp. 105/106. The factor of 2 on the right-hand side of Eqs. (2.1), (2.2), and (2.3) on p. 105 should be dropped. On p. 106, the factor of 2 in Eq. (2.5) and in the first centered equation after that should also be dropped. In Proposition 4, the first equation in braces should have the 3 replaced by 2 , and the first plus sign replaced by a minus sign. The second equation in the brace should have the 2 replaced by 1 . The limits at the end of Proposition 4 should be adjusted accordingly. In the proof of the proposition, the two places that $2 \lambda$ appear should be replaced by $\lambda$.

The authors should like to thank Yoram Last for pointing out these errors.

Communicated by A. Jaffe 